\title{
CONSTRUCTIVE-FUNCTIONAL ANALYSIS AND SIZING OF HYDRAULIC FILTERS
}

\author{
Nicușor BAROIU ${ }^{1}$, Georgiana Alexandra MOROȘANU ${ }^{1}$
}

${ }^{1}$ Department of Manufacturing Engineering, "Dunărea de Jos” University of Galați, România email: Alexandra.Costin@ugal.ro

\begin{abstract}
The reliability of hydrostatic installations depends on the low level of impurities in an environment with pressure at an allowable value, which avoids an accentuated wear of the individual elements of the installations. Hydraulic filters are elements that have a role in protecting the hydrostatic installations from the particles that are found in the hydraulic oil. The paper aims to present some of the elements underlying the construction of hydraulic environment filtration equipment, certain conditions that are imposed on them, as well as the main problems related to the calculation and sizing of filters.
\end{abstract}

KEYWORDS: filters, filtering surface, filter elements, sizing of filters.

\section{INTRODUCTION}

The good functioning of the hydraulic systems, but also the maintenance of the initial performances, depends to a large extent on the degree of purity of the hydraulic fluid. Impurities cause, by their nature, the erosion of the elements in contact, the gripping of the moving elements, the clogging of the orifices, as well as the chemical degradation of the engine agent [1-3].

Figure 1 shows several types of filters.

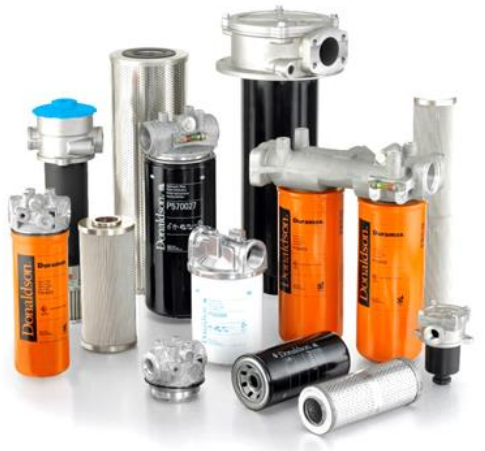

Fig. 1. Types of filters [4]

Filters found in hydrostatic installations, Figure 2, are subject to certain conditions [1]:

- to have the capacity to retain impurities with different dimensions;

- to present the possibility of fast and periodic cleaning of the filter element;

- to allow easy replacement of the filter element;
- to present the possibility to control the operation of the filters;

- to ensure the protection of the installation in case of clogging of the filter element.

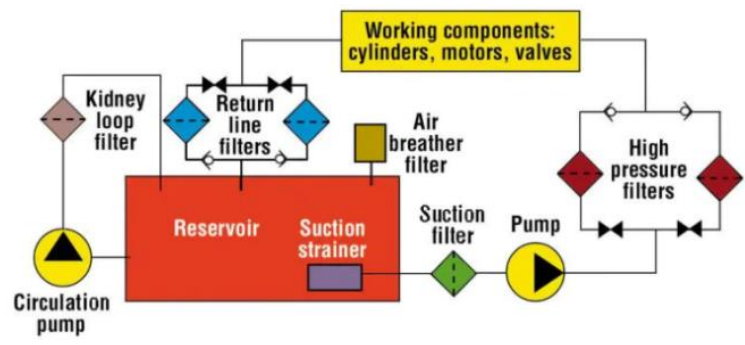

Fig. 2. Filter configuration [5]

The main element in the construction of filters is the filter element, while the filtering method consists in forcing the agent to pass through it.

The classification of filters is shown in Figure 3.

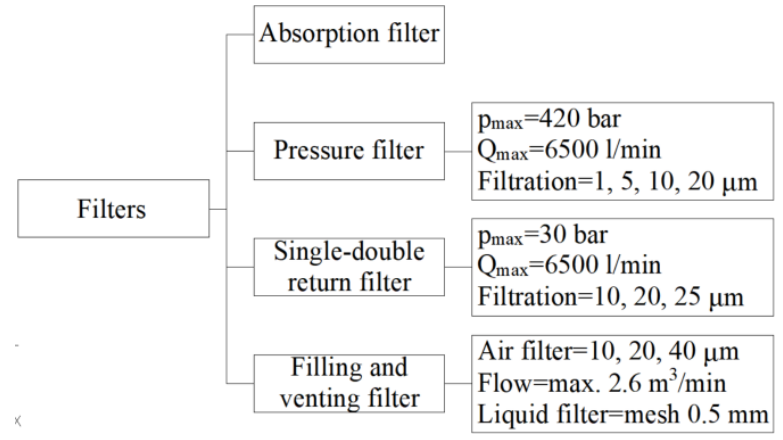

Fig. 3. The classification of filters [6] 
Impurity classes indicate how many particles of a certain size are contained in $100 \mathrm{ml}$ of hydraulic fluid. The determination of the impurity class is done by counting and classifying the size of the impurity particles. Starting with a concentration of impurities of about $10 \mathrm{mg} /$ liter or in a very strong liquid disorder, the degree of impurity can only be ascertained by determining the weight of the impurities by gravimetric analysis $[7,8]$.

In a hydraulic system, the most sensitive components to impurities are servo valves and proportional valves. Therefore, they determine the total impurity classes of the hydraulic oil and the required filtration fineness.

\section{STRUCTURE OF IMPURITY}

\section{CLASSES}

ISO 4406:2021 (Hydraulic fluid power-FluidsMethod for coding the level of contamination by solid particles) regulates the classification systems of impurity classes in hydraulic oils.

Figure 4 indicates the particle sizes on the $X$-axis and on the $Y$-axis, the number of particles is written and they are divided into classes 1-20. The line drawn on the diagram describes the distribution of particles in the hydraulic oil. The ascending slope of the line is determined by the entry of the particle size of $5 \mu \mathrm{m}$ and $15 \mu \mathrm{m}$.

By determining the class number for $5 \mu \mathrm{m}$ particles and $15 \mu \mathrm{m}$ particles, the particle distribution line is described.

For example, for servo valves and proportional valves, the following oil purity is required: servo valves $(13 / 10)$ - red curve; proportional valves (17/14) - blue curve [7].

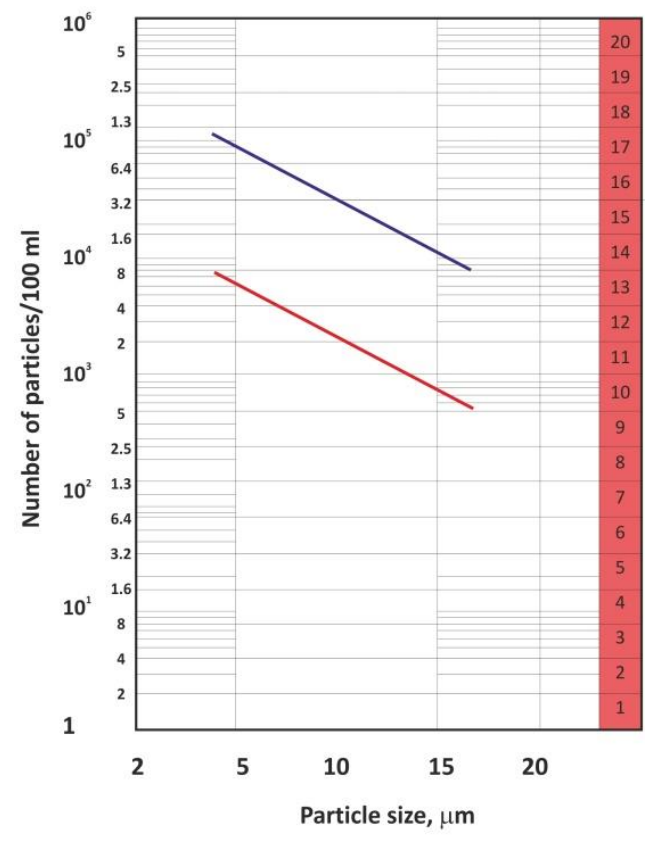

Fig. 4. Purity class structure according to ISO 4406:2021
Thus, the individual particle sizes are comprised of 5 domains. For each domain, a maximum particle quota is indicated for each class, Table 1.

For example, the purity required for oil, according to the NAS 1638 standard, developed in order to define the levels of contamination in the aerospace sector [7], is servo valves: from 4 to 6 (red domain), respectively proportional valves: from 8 to 9 (blue domain).

Table 1. Structure of purity classes (NAS 1638)

\begin{tabular}{|c|c|c|c|c|c|}
\hline \multicolumn{6}{|c|}{$\begin{array}{c}\text { Maximum number of impurity particles in } 100 \\
\text { ml of hydraulic fluid at particle size }\end{array}$} \\
\hline \multirow{2}{*}{ Class } & \multicolumn{5}{|c|}{$\boldsymbol{\mu m}$} \\
\cline { 2 - 6 } & $5-11$ & $15-25$ & $25-50$ & $50-100$ & $>100$ \\
\hline 00 & 125 & 22 & 4 & 1 & 0 \\
\hline 0 & 250 & 44 & 8 & 2 & 0 \\
\hline 1 & 500 & 89 & 16 & 3 & 1 \\
\hline 2 & 1000 & 178 & 32 & 6 & 1 \\
\hline 3 & 2000 & 356 & 63 & 11 & 2 \\
\hline 4 & 4000 & 712 & 126 & 22 & 4 \\
\hline 5 & 8000 & 1425 & 253 & 45 & 8 \\
\hline 6 & 16000 & 2850 & 506 & 90 & 16 \\
\hline 7 & 32000 & 5700 & 1012 & 180 & 32 \\
\hline 8 & 64000 & 11400 & 2025 & 360 & 64 \\
\hline 9 & 128000 & 22800 & 4050 & 720 & 128 \\
\hline 10 & 256000 & 45600 & 8100 & 1440 & 256 \\
\hline 11 & 512000 & 91200 & 16200 & 2880 & 512 \\
\hline 12 & 1024000 & 182400 & 32400 & 5760 & 1024 \\
\hline
\end{tabular}

A parameter, called the $\beta_{x}$ value, serves as a standard for restraint speed of impurities (filtration fineness). In general, the $\beta_{x}$ value always refers to particles that are larger than the observed $X$ particle size.

Impurity particles counted before the filter element of a certain particle size $X$ are divided by the impurity particles counted after the filter element (same particle size $X$ at the same differential pressure counted at the same time point).

The non-dimensional number obtained represents the value of $\beta_{x}$. The representation of the deposition of impurity particles through the filter elements is shown in Figure 5.

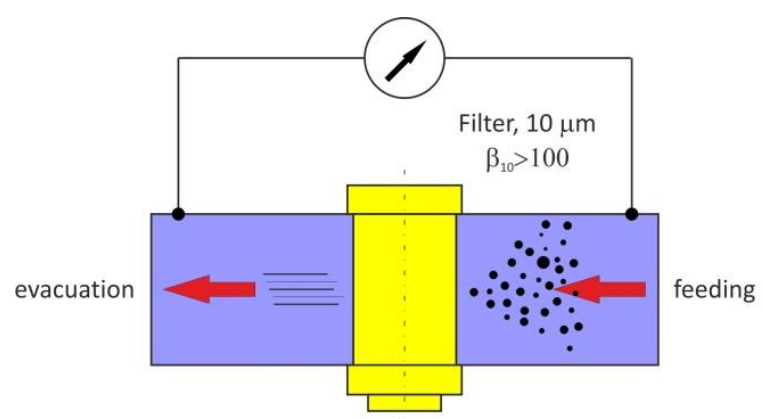

Fig. 5. The representation of the deposition of impurity particles through the filter elements 
Example of a number of measured particles: - feeding flow: 10000 particles in $100 \mathrm{ml}$; - evacuation flow: 100 particles in $100 \mathrm{ml}$.

$$
\beta_{3}=\frac{n_{z \text { feeding flow }}}{n_{z \text { evacuation flow }}}=\frac{10000}{100}=100,
$$

$\beta_{3}=100=99 \%$ (also called separation degree).

The indication of the $\beta_{x}$ value indicates the precipitation behavior (yield) of the filter element. The advantage is that the domain between $90 \%$ and $100 \%$ precipitation degree can be greatly expanded. Once the $\beta_{x}$ value is defined, taking into account the differential pressure that is formed, it is possible to compare the indications of filter fineness according to different filter materials.

Definitions are accepted for the filter fineness [9]: 1. nominal filtration fineness - no usual $\beta_{x}$ values are established; this means that only part of the filterable impurities with an optimal filter is removed by filtration. For $\beta_{x} \geq 20$ - this corresponds to a precipitation degree of about $95 \%$;

2. absolute filtration fineness - from a value of $\beta_{x} \geq 100$ or a precipitation degree of $99 \%$, the filtration fineness is designated as absolute retention speed.

A hydraulic filter element represents a device that is designed to eliminate contaminants in fluids [10].

In most cases, these contaminants come from: - recirculation of hydraulic fluid through tanks; - internal generation of particles in the components of the hydraulic system, such as pumps and motors.

\section{CONSTRUCTIVE ELEMENTS OF FILTERS}

The filter elements are mounted in the housing, the construction which depends on the nature, size, and shape of the filter element and the destination of the filter. Therefore, some of the most used filter elements are [1]:

a. filter elements with wire mesh (Figure 6) have a filtration fineness in the range $60 \div 500 \mu \mathrm{m}$.

It is presented in the form of a cylindrical cartridge or superimposed rings, made of brass or copper wire, with a number of $3000 \div 20000$ per $\mathrm{cm}^{2}$.

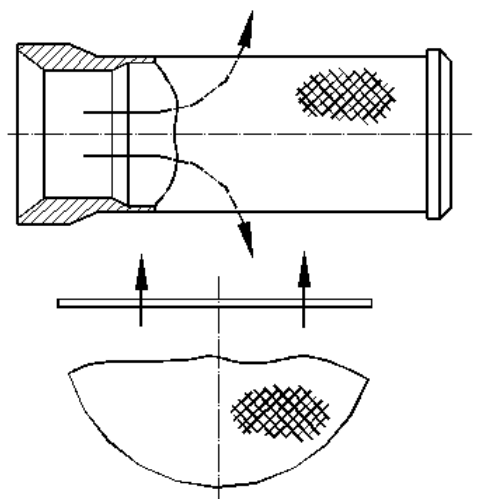

Fig. 6. Filter element with wire mesh b. filter elements with slats (Figure 7) have a filtration fineness of $16 \div 250 \mu \mathrm{m}$.

They consist of the discs (2) and the spacer slats (3), arranged intercalated on an axis (1). The thickness of the spacer slats ensures gaps of $\delta_{1}=0.005-0.1 \mathrm{~mm}$, the thickness $\delta$ of the discs being $0.1 \div 0.2 \mathrm{~mm}$.

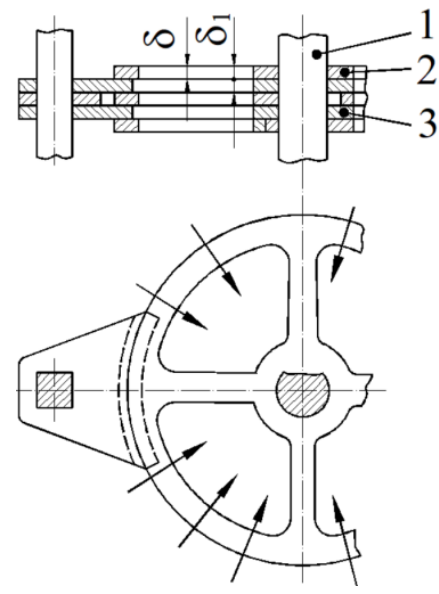

Fig. 7. Filter elements with slats

c. paper filter element has a filtering fineness between 10 and $25 \mu \mathrm{m}$.

It is presented in the form of overlapping rings or cylinders made of embossed (folded) paper, thus increasing the contact surface between the fluid and the filter element (filter surface);

$d$. sintered powder filter elements have a filtration fineness between 2 and $10 \mu \mathrm{m}$, retaining impurities of any kind, with dimensions of $3 \div 5 \mu \mathrm{m}$, in the proportion of $100 \%$.

It is presented in the form of a glass, cylinder, Figure 8 , or overlapping rings, obtained by pressing in specific shapes at high pressures $(1000 \div 3000 \mathrm{bar})$ of bronze, steel, ceramic materials, followed by ripening (sintering) in the oven.

The size of the pores (1) obtained by sintering represents $10 \%$ of the diameter of the initial powder granules.
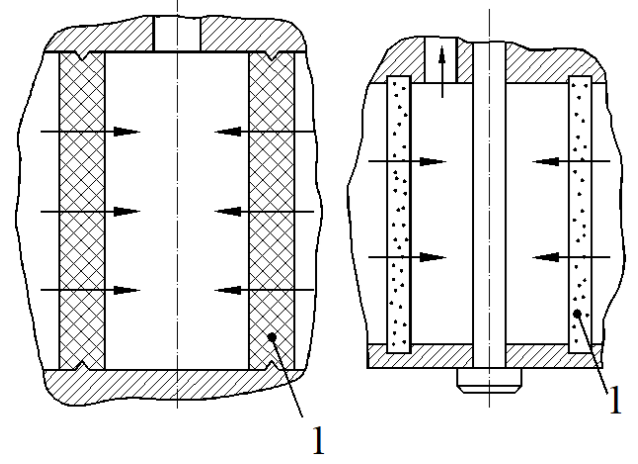

Fig. 8. Sintered powder filter element

e. filter elements with magnets - retain particles with magnetic properties; permanent magnets (ceramics) or electromagnets are used.

The magnet (1), Figure 9, is protected from direct contact with the fluid through the coating (2). 


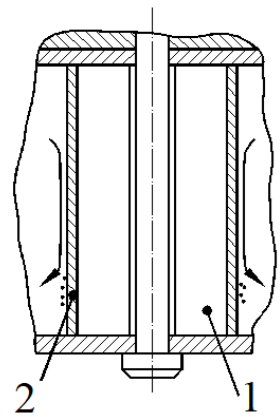

Fig. 9. Filter element with magnets

g. electrostatic filter elements, Figure 10 - used to electrify impurity particles (1) in an electrostatic field created by two electrodes (2), connected at a voltage of $300 \div 600 \mathrm{~V}$.

The electrodes attract and retain the electrified impurities, being covered with a ceramic material (3) in order to avoid their neutralization.

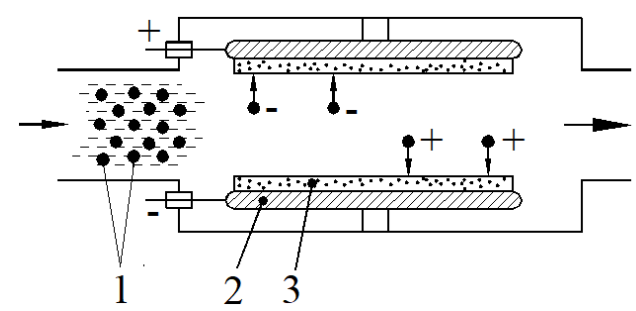

Fig. 10. Electrostatic filter element

h. centrifugal filter elements - filter by throwing (centrifuging) the particles of impurities on the wall of the housing under the action of the centrifugal force created by rotating the axis with high speed. The fineness of the filtration depends on the rotational speed, which can reach a value of $5 \mu \mathrm{m}$ for a speed of $20000 \mathrm{rpm}$. Such constructions are used to filter important fluid flows.

The construction of a filter with filter element with wire mesh is presented in Figure 11.

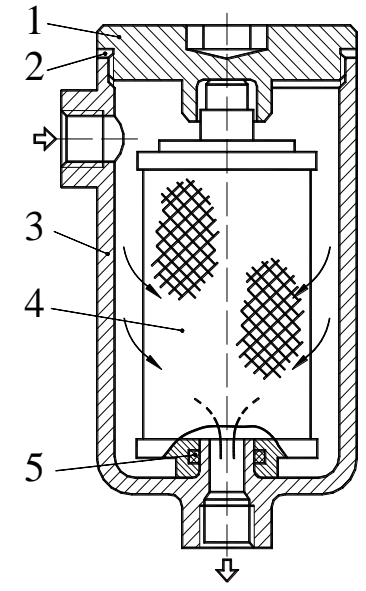

Fig. 11. Filter with wire mesh: 1 - lid; 2 - gasket; 3 - body; 4 - filter element; 5 - sealing gasket.

In the simple version, they have a single mesh and in the combined version they have several meshes.
Periodic cleaning of the filter involves the removal and unclogging of the filter element or its replacement with a new one.

Usually, in hydraulic installations, magnetic filters, Figure 12, are used as complementary filters. They only separate metallic impurities with magnetic properties. When passing through the filter, the fluid is forced to flow through the collecting rings (5), so that the respective impurities are attracted and retained by the magnetic core (4).

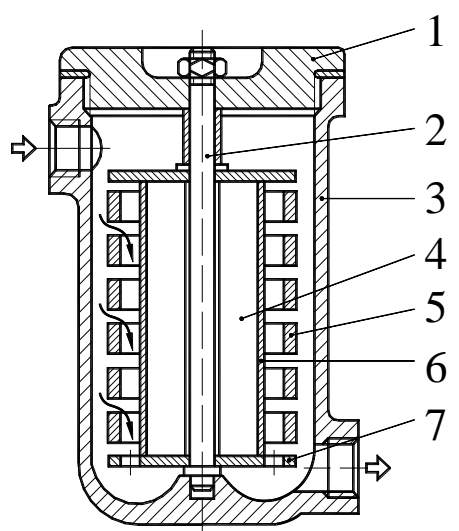

Fig. 12. Magnetic filter: 1 - lid; 2 - fixing rod; 3 - body; 4 - permanent magnet; 5 - collecting rings; 6 - protective coating for magnet; 7 - fixing thaler.

\section{FILTER INSTALLATION SOLUTIONS}

The installation of filters in hydraulic circuits can be done in several variants [1]:

a. suction filter or low pressure filter, Figure 13.a;

b. high-pressure filters, Figure 13.b;

c. filter on the exhaust pipe from the engine or return filter, Figure 13.c;

d. return filter with protection, Figure 13.d;

e. filter on a special secondary circuit, Figure 13.e.

A suction filter or low pressure filter, Figure 13.a, is usually made of mesh type with a fineness of $100 \div 200 \mu \mathrm{m}$. It has the role of protecting the pump and must have a pressure drop $\Delta p$ as low as possible, in order to avoid the cavitation phenomenon of the pump.
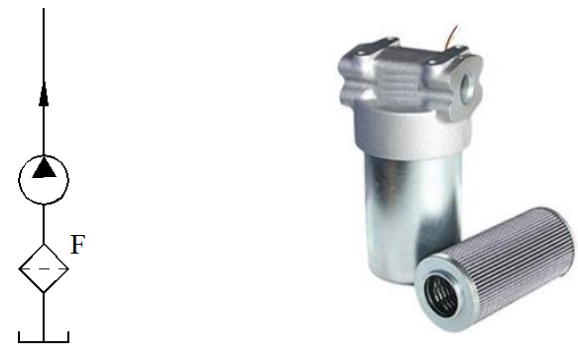

Fig. 13.a. Filter installation solutions: suction filter or low pressure filter [11]

Pressure pipe filter or high pressure filter, Figure 13.b, is usually made of mesh or slats type but 
especially of sintered powders with a filtration fineness of $2 \div 10 \mu \mathrm{m}$.

This filter aims to protect precision hydraulic devices such as speed regulators, servo valves, etc.
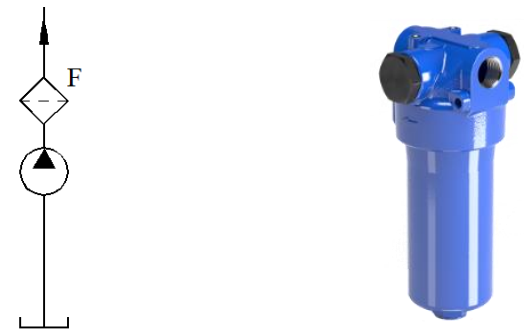

Fig. 13.b. Filter installation solutions: pressure pipe filter or high pressure filter [12]

The filter solution on the exhaust pipe from the engine or return filter, Figure 13. c, is often used as the position in the circuit and allows them to drop pressure more than the suction filters. They have the disadvantage of worsening the energy balance. They are usually of mesh or paper type, with a fineness of $10 \div 40 \mu m$
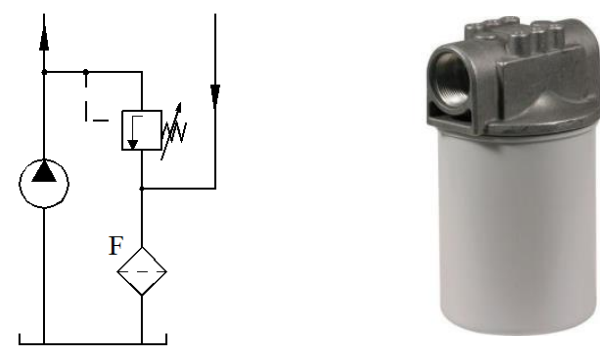

Fig. 13.c. Filter installation solutions: filter on the exhaust pipe from the engine or return filter [13]

The solution of the return filter with protection, Figure 13.d, also involves the installation of the filter on the evacuation pipe from the engine. When the filter is clogged, the increase in pressure drop could damage it and can be prevented by opening the bypass valve $(S)$. As the filter is switched off in this case, the system remains unprotected from this point of view.

For this, it is recommended to use special devices to indicate the clogging of the filter element that warns and indicates the need for intervention in order to clean or change the filter element.
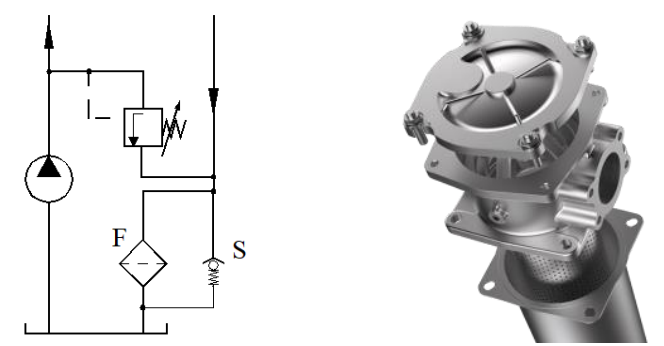

Fig. 13.d. Filter installation solutions: return filter with protection [8]

The solution of the filter on a special secondary circuit, Figure 13.e, involves the installation of a return filter $(F)$ on the pump circuit $(P 2)$ which permanently recirculates the liquid from the tank $(R)$ in order to purify it.

This is the case for complex installations, but the solution does not eliminate the need for the main circuit of the pump $(P 1)$ to have its own filters.

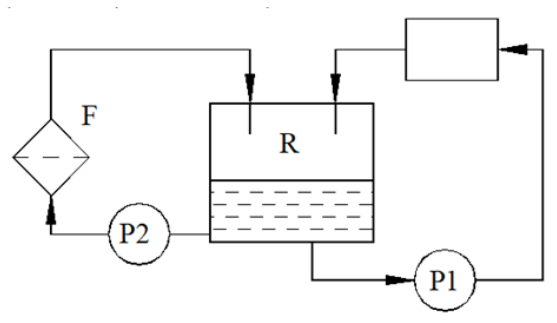

Fig. 13.e. Filter installation solutions: filter on a special secondary circuit

\section{FILTER SIZING}

The calculation of the filters consists in determining the filtering surface $S$ of the filter element, using the relation [1]:

$$
S=0,01 \frac{Q}{\alpha \cdot \Delta p} \cdot \eta\left[\mathrm{cm}^{2}\right],
$$

where:

- $Q$ is the fluid flow in $l / \mathrm{min}$;

- $\eta$ - dynamic viscosity of the fluid, in $10^{-3} \mathrm{~N} \mathrm{sec} / \mathrm{m}^{2}$.

- $\alpha$ - coefficient of the specific capacity of the fluid to pass through the filter element, in $l / \mathrm{cm}^{2}$.

- $\Delta p$ - pressure drop in the filter, in bar.

The value of the coefficient $\alpha$ depends on the type of filter element, Table 2 .

Table 2. The value of the coefficient $\alpha$ depending on

\begin{tabular}{|c|c|}
\multicolumn{2}{|c}{ the type of filter element } \\
\hline Filter element type & $\boldsymbol{\alpha}$ \\
\hline Cotton & $\alpha_{1}=0.009$ \\
\hline Felt & $\alpha_{2}=0.015$ \\
\hline Wire mesh & $\alpha_{3}=0.05$ \\
\hline Slats with a thickness of $0.05 \div 0.08 \mathrm{~mm}$ & $\alpha_{4}=0.08$ \\
\hline
\end{tabular}

Therefore, four types of filtering surface $S$ of the filter element will be calculated according to parameter $\alpha$ :

$$
\begin{aligned}
& S_{1}=0,01 \frac{Q}{\alpha_{1} \cdot \Delta p} \cdot \eta\left[\mathrm{cm}^{2}\right], \quad S_{2}=0,01 \frac{Q}{\alpha_{2} \cdot \Delta p} \cdot \eta\left[\mathrm{cm}^{2}\right], \\
& S_{3}=0,01 \frac{Q}{\alpha_{3} \cdot \Delta p} \cdot \eta\left[\mathrm{cm}^{2}\right], \quad S_{4}=0,01 \frac{Q}{\alpha_{4} \cdot \Delta p} \cdot \eta\left[\mathrm{cm}^{2}\right],
\end{aligned}
$$

The values for a flow $Q$ necessary for the operation of a hydrostatic motor will be chosen arbitrarily and, depending on one of the installation solutions presented in Figures 13.a $\div 13$.e, the surface of the filter element will be calculated. 
Also, the dynamic viscosity of the fluid, $\eta$, and the pressure drop in the filter, $\Delta p$, will be chosen arbitrarily, considering that the parameter $\eta$ will be between the limits $2.5 \div 5 \mathrm{daN} \cdot \mathrm{s} / \mathrm{m}^{2}$ and $\Delta p$ between $0.5 \div 3$ bar.

Therefore, the input data for the calculation of the filtering surface $S$ are presented in Table 3.a and Table 3.b. The results for data $1 \div 10$ and $11 \div 20$ corresponding to Table 3.a and Table 3.b are presented graphically in Figure 14 and Figure 15.

Table 3.a. The input data for the calculation of the filtering surface $S$

\begin{tabular}{|c|c|c|c|}
\hline No. & $\mathbf{Q}[\mathbf{l} / \mathbf{m i n}]$ & $\boldsymbol{\eta}\left[\mathbf{d a N} \cdot \mathbf{s} / \mathbf{m}^{\mathbf{2}}\right]$ & $\Delta \mathbf{p}[\mathbf{b a r}]$ \\
\hline 1 & 56 & 2.8 & 1.3 \\
\hline 2 & 60 & 4 & 2.1 \\
\hline 3 & 52 & 3.2 & 0.5 \\
\hline 4 & 48 & 3.6 & 2 \\
\hline 5 & 50 & 3.1 & 1 \\
\hline 6 & 58 & 2.9 & 0.7 \\
\hline 7 & 40 & 3.3 & 1.4 \\
\hline 8 & 45 & 3.4 & 1.6 \\
\hline 9 & 54 & 3.7 & 1.7 \\
\hline 10 & 47 & 3.5 & 0.6 \\
\hline
\end{tabular}

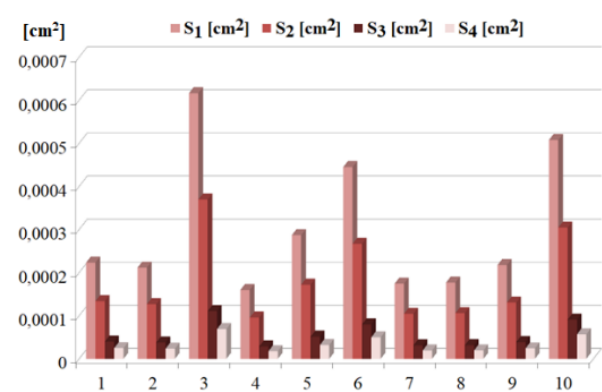

Fig. 14. The results of filtering surfaces calculation, $S$ for data $1 \div 10$ corresponding to Table 3.a

Table 3.b. The input data for the calculation of the filtering surface $S$

\begin{tabular}{|c|c|c|c|}
\hline No. & $\mathbf{Q}[\mathbf{l} / \mathbf{m i n}]$ & $\boldsymbol{\eta}\left[\mathbf{d a N} \cdot \mathbf{s} / \mathbf{m}^{\mathbf{2}}\right]$ & $\Delta \mathbf{p}[\mathbf{b a r}]$ \\
\hline 11 & 42 & 3.8 & 0.9 \\
\hline 12 & 49 & 2.6 & 1.8 \\
\hline 13 & 57 & 3 & 2.5 \\
\hline 14 & 53 & 2.7 & 2.2 \\
\hline 15 & 41 & 2.3 & 0.8 \\
\hline 16 & 59 & 3.9 & 2.4 \\
\hline 17 & 51 & 2.5 & 1.5 \\
\hline 18 & 46 & 3 & 1.2 \\
\hline 19 & 55 & 3.2 & 1.9 \\
\hline 20 & 43 & 2.7 & 2.3 \\
\hline
\end{tabular}

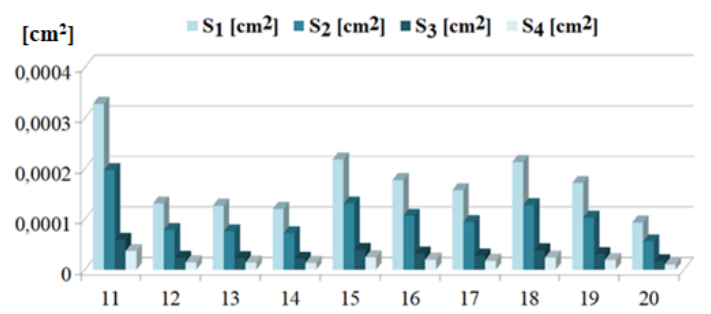

Fig. 15. The results of filtering surfaces calculation, $S$ for data $11 \div 20$ corresponding to Table $3 . b$

\section{CONCLUSIONS}

The choice of the material of the filter element is the basis of the construction of the filtering equipment of the hydraulic environment and has a specific influence on the problems related to the calculation and sizing of the filters.

The paper presents a way of calculating the surface of the filter element, by arbitrarily choosing the values for a flow $Q$ necessary for the operation of a hydrostatic motor. Depending on this parameter, the flow rate $Q$, and the permissible pressure drop $\Delta p$, a filter of a certain type will be chosen from the catalog of manufacturing companies.

\section{ACKNOWLEDGEMENTS}

This work is supported by the project ANTREPRENORDOC, in the framework of Human Resources Development Operational Programme 2014-2020, financed from the European Social Fund under the contract number 36355/23.05.2019 HRD OP /380/6/13 - SMIS Code: 123847.

\section{REFERENCES}

[1] Baroiu, N., Vișan, D., Ciocan, O.D., Hidrostatică și pneumatică tehnologică - Indrumar pentru laborator - format electronic, Ed. Academica, ISBN 978-606-606-007-3, 2018;

[2] Hujo, L., Jablonický, J., Markovic, J., Tulík., J., Simikic, M., Zastempowski, M., Janoušková, R., Design of Laboratory Test Equipment for Automotive Oil Filters to Evaluate the Technical Life of Engine Oil, Appl. Sci. 11:483, 2021, https://doi.org/10.3390/app11020483;

[3] Momin, T., Chandrasekar, R.P., Balasubramanian, S., Junaid Basha, A.M., Design and Analysis of High Pressure Hydraulic Filter for Marine Application, IOP Conf. Series: Materials Science and Engineering 197, 012050, doi:10.1088/1757899X/197/1/012050, 2017;

[4] Donaldson, Assemblies, https://www.donaldson.com/enbe/engine/filters/products/hydraulics/assemblies/;

[5] Hydraulics \& Pneumatics, Which hydraulic filters are most effective?

https://www.hydraulicspneumatics.com/ technologies/hydraulicfilters/article/21883277/which-hydraulic-filters-are-most-effective;

[6] Schmitt, A., The Hydraulic Trainer: Instruction and Information on Oil Hydraulics, Mannesmann Rexroth, 1980;

[7] Dorr, H., Ewald, R., Hutter, J., Kretz, D., Liedhegener, F., Schmitt, A., Hydraulic Trainer, Volume 2: Proportional and Servo Valve Technology Hardcover, ISBN 3-8023-0898-0, 1986;

[8] Exner, H., Freitag, R., Geis, H., Lang, R., Oppolzer, J., Schwab, P., Sumpf, E., Der Hydraulik Trainer - Grundlagen und Komponenten der Fluidtechnik Hydraulik, Der Hydraulik Trainer Band 1, ISBN: 3802306198, 1991;

[9] Gebhardt, N., Weber, J., Hydraulik - Fluid-Mechatronik: Grundlagen, Komponenten, Systeme, Messtechnik und virtuelles Engineering, Springer-Verlag, ISBN 978-3662-60663-6, 2020

[10] Indiamart, Hydraulic filter, https://www.indiamart.com/ proddetail/hydraulic-filter-5757787248.html;

[11] Hebei Huilong Hydraulic Machinery Co., Ltd., Low pressure filters, http://filter-manufacture.com/1-3-low-pressurefilters.html;

[12] MP Filtri, In-line filters > 80 bar (high pressure), https://www.mpfiltri.com/prodotti/hydraulic-filtration-1/in-linefilters-80-bar-high-pressure-11/;

[13] Indiamart, Hydraulic return filter, https://www.indiamart. com/proddetail/hydraulic-return-filter-14096680133.html;

[14] UFI Filters, Return filters,

https://www.ufihyd.com/products/ return-filters/. 